\title{
SCIENTIFIC REP

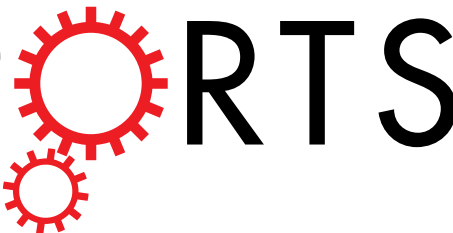 \\ OPEN \\ Author Correction: Remora cranial vein morphology and its functional implications for attachment
}

Brooke E. Flammang ${ }^{1}{ }^{1} \&$ Christopher P. Kenaley ${ }^{2}$

Correction to: Scientific Reports https://doi.org/10.1038/s41598-017-06429-z, published online 19 July 2017

Christopher P. Kenaley was omitted from the author list in the original version of this Article. This has been corrected in the PDF and HTML versions of the Article.

The Acknowledgements section now reads:

BEF wishes to thank E.J. Anderson, J.H. Nadler, M. Beckert, A.P. Summers, and G.V. Lauder for their encouraging discussions. A.P. Russell offered tremendous insight that greatly improved the quality of this manuscript. K. Hartel and A. Williston (Museum of Comparative Zoology, Harvard University) generously loaned specimens for comparative observation. S. Crofts, C. Crawford, K. Gamel, L. Winn, and A. Tam assisted in the dye injection experiments. K. Suri and K. Stanchak assisted in several perfusion experiments and CT scans. D. Benetti and J. Fiorentino (RSMAS, University of Miami) provided the cobia. B. Ache and B. Walters (MicroPhotonics, Inc.) assisted in $\mu \mathrm{CT}$ scanning.

The Author Contributions section now reads:

BEF discovered the anatomy, conceived and conducted the experiments, analysed the data, developed the functional theory, prepared the figures, and wrote the manuscript. CPK provided the contrast agent and operated the CT scanner for the experiment that resulted in Figure 1.

(i) Open Access This article is licensed under a Creative Commons Attribution 4.0 International License, which permits use, sharing, adaptation, distribution and reproduction in any medium or format, as long as you give appropriate credit to the original author(s) and the source, provide a link to the Creative Commons license, and indicate if changes were made. The images or other third party material in this article are included in the article's Creative Commons license, unless indicated otherwise in a credit line to the material. If material is not included in the article's Creative Commons license and your intended use is not permitted by statutory regulation or exceeds the permitted use, you will need to obtain permission directly from the copyright holder. To view a copy of this license, visit http://creativecommons.org/licenses/by/4.0/.

(C) The Author(s) 2018 